\title{
Communication intercellulaire et infections
} microbiennes / Intercellular communication and microbial infections

Centre interdisciplinaire de recherche en biologie (CIRB)

\section{Guy Tran Van Nhieu}

\section{OpenEdition \\ Journals}

Édition électronique

URL : https://journals.openedition.org/annuaire-cdf/16163

DOI : 10.4000/annuaire-cdf.16163

ISBN : 978-2-7226-0572-5

ISSN : 2109-9227

Éditeur

Collège de France

\section{Édition imprimée}

Date de publication : 30 décembre 2020

Pagination : 664-666

ISBN : 978-2-7226-0516-9

ISSN : 0069-5580

\section{Référence électronique}

Guy Tran Van Nhieu, " Communication intercellulaire et infections microbiennes / Intercellular communication and microbial infections ", L'annuaire du Collège de France [En ligne], 118 | 2020, mis en ligne le 01 avril 2021, consulté le 22 août 2022. URL : http://journals.openedition.org/annuaire-cdf/ 16163 ; DOI : https://doi.org/10.4000/annuaire-cdf.16163 


\title{
COMMUNICATION INTERCELLULAIRE ET INFECTIONS MICROBIENNES / INTERCELLULAR COMMUNICATION AND MICROBIAL INFECTIONS
}

\author{
Responsable: Guy Tran VAN NHIEU
}

\section{RECHERCHE}

Au cours des deux dernières décennies, il est devenu évident que, comme décrit pour les cellules eucaryotes, la sous-localisation des protéines dans les bactéries joue un rôle clé dans leur fonction ou leur régulation spatiotemporelle [1, 2]. Cependant, contrairement aux cellules eucaryotes, les bactéries ne contiennent pas de membranes intracellulaires délimitant les compartiments internes. Ainsi, la localisation des protéines bactériennes repose sur d'autres mécanismes encore mal compris, dont certains sont spécifiques à certaines espèces bactériennes. En particulier, le mécanisme sous-jacent à la localisation de protéines cytoplasmiques «fonctionnelles» au niveau de pôles bactériens a fait l'objet de débats, car les agrégats amorphes sont également décrits comme étant exclus des nucléoïdes des cellules bactériennes pour s'accumuler aux pôles [3]. La localisation des agrégats de protéines dans une région dépourvue de nucléoïde peut s'expliquer par un encombrement moléculaire et une entrave à la diffusion. Ce processus est appelé « occlusion par le nucléoïde », terme utilisé à l'origine sur la base de l'observation que le nucléoïde empêche la formation de l'anneau de division conduisant à la septation bactérienne [4]. De petits agrégats de protéines alimentent la croissance amorphe de gros agrégats, expliquant leur accumulation préférentielle au niveau d'un pôle ou bipolaire [3]. Cependant, outre les agrégats, des protéines «fonctionnelles » peuvent également s'accumuler au niveau des pôles bactériens, caractéristique essentielle à leur fonction. Deux mécanismes principaux ont été proposés pour la localisation polaire. L'un est un mécanisme de diffusion-capture qui ne se limite pas aux pôles bactériens, mais peut également expliquer la localisation des protéines sur d'autres sites. Cependant, dans de nombreux cas, comme pour les protéines de division cellulaire, la nature du déterminant de localisation primaire polaire est restée indéterminée [5]. La localisation polaire peut être déterminée par la capacité d'une protéine à détecter l'augmentation de la courbure des lipides membranaires négatifs au niveau des pôles bactériens [2]. En variante, la liaison des protéines périphériques à la cardiolipine, un phospholipide qui s'accumule de préférence dans les membranes incurvées négativement au niveau du pôle bactérien, pourrait conduire à la localisation polaire $[1,2]$. Le deuxième mécanisme proposé pour la localisation polaire est l'exclusion de la protéine native formant de grandes structures oligomères par le nucléoïde [2,3]. Diverses protéines, notamment les autotransporteurs, ont montré une localisation unipolaire [6]. La protéine de surface cellulaire Shigella IcsA responsable de la motilité intracellulaire à base d'actine représente probablement le paradigme de la localisation unipolaire chez les bactéries reliées à Escherichia coli. La localisation polaire IcsA résulte de sa localisation intra-bactérienne au pôle avant la sécrétion [7]. Le mécanisme sousjacent à la localisation unipolaire IcsA n'est pas complètement compris. Le chaperon DnaK a été identifié comme étant requis pour la polarisation IcsA dans un écran de génome large de protéines fusionnées à la protéine fluorescente verte (GFP), mais la 
façon dont ce chaperon contrôle la localisation polaire IcsA n'a pas été clarifiée [8]. Dans des travaux antérieurs, nous avions montré qu'avant le contact avec les cellules, le composant translocon IpaC de Shigella de type III se localisait également à un pôle bactérien et que cette localisation polaire déterminait la sécrétion de type III de type polaire lors de l'invasion des cellules hôtes par Shigella [9]. La localisation unipolaire de IpaC parallèle celle d'IcsA et n'est pas liée à l'agrégation de protéines car les protéines au pôle s'échangent avec les protéines du reste du corps bactérien. Fait intéressant, ces expériences de chasse ont indiqué que le pool d'IpaC polaire est soumis à un contrôle protéolytique à l'état d'équilibre [9]. Nous avons utilisé une approche biochimique pour identifier les protéines impliquées dans la polarisation de IpaC. Nos travaux récents montrent que le chaperon DnaK (HSP70) contrôle la localisation unipolaire du substrat de sécrétion de Shigella IpaC de type III [10]. Tout en empêchant la formation d'agrégats létaux IpaC, DnaK favorise l'incorporation d'IpaC dans de larges complexes dynamiques (LCDs) restreints au pôle bactérien par occlusion par le nucléoïde [10]. Contrairement aux polymères et aux agrégats stables, les LCDs sont réversibles et indiquent que l'occlusion de nucléoïde s'applique également aux réseaux formés par des interactions transitoires. Une analyse par recouvrement de fluorescence après photoblanchiment (FRAP) a montré que les complexes DnaK-IpaC s'échangent entre pôles opposés et que les LCDs incorporent des substrats immatures dans un processus assisté par la machine DnaKJE [10]. Ces découvertes révèlent un rôle essentiel pour les LCDs en tant que réservoirs de substrats fonctionnels de la chaperone DnaK, pouvant être rapidement mobilisés pour la sécrétion déclenchée lors du contact bactérien avec les cellules hôtes.

\section{Références citées}

[1] KiRKPATRICK C.L. et ViollieR P.H., « Poles apart: Prokaryotic polar organelles and their spatial regulation », Cold Spring Harbor Perspectives in Biology, vol. 3, $\mathrm{n}^{\mathrm{o}} 3$, 2011, DOI : 10.1101/cshperspect.a006809.

[2] LALOUX G. et JACOBS-WAGNER C., «How do bacteria localize proteins to the cell pole? », Journal of Cell Science, vol. 127, 2014, p. 11-19, DOI : 10.1242/jcs.138628.

[3] Winkler J., Seybert A., König L., Pruggnaller S., Haselmann U., SourJik V., WeisS M., Frangakis A.S., Mogk A. et Bukau B., «Quantitative and spatio-temporal features of protein aggregation in Escherichia coli and consequences on protein quality control and cellular ageing », The EMBO journal, vol. 29, no 5, 2010, p. 910-923, DOI : 10.1038/ emboj.2009.412.

[4] Wu L.J. et ERRINGTON J., « Nucleoid occlusion and bacterial cell division », Nature Reviews. Microbiology, vol. 10, no 1, 2011, p. 8-12, DOI : 10.1038/nrmicro2671.

[5] Haeusser D.P. et Margolin W., «Splitsville: Structural and functional insights into the dynamic bacterial Z ring », Nature Reviews. Microbiology, vol. 14, n 5, 2016, p. 305-319, DOI : $10.1038 /$ nrmicro.2016.26.

[6] Jain S., van Ulsen P., Benz I., Schmidt M.A., Fernandez R., Tommassen J. et GOLDBERG M.B., «Polar localization of the autotransporter family of large bacterial virulence proteins », Journal of Bacteriology, vol. 188, $\mathrm{n}^{\circ}$ 13, 2006, p. 4841-4850, DOI : 10.1128/ JB.00326-06.

[7] Charles M., Pérez M., Kobil J.H. et Goldberg M.B., «Polar targeting of Shigella virulence factor IcsA in Enterobacteriacae and Vibrio », Proceedings of the National Academy of Sciences of the United States of America, vol. 98, n 17, 2001, p. 9871-9876, DOI : 10.1073/ pnas. 171310498. 
[8] Janakiraman A., Fixen K.R., Gray A.N., Niki H. et Goldberg M.B., «A genomescale proteomic screen identifies a role for DnaK in chaperoning of polar autotransporters in Shigella », Journal of Bacteriology, vol. 191, no 20, 2009, p. 6300-6311, DOI : 10.1128/ JB.00833-09.

[9] Jaumouillé V., Francetic O., Sansonetti P.J. et Tran Van Nhieu G., « Cytoplasmic targeting of IpaC to the bacterial pole directs polar type III secretion in Shigella », The EMBO Journal, vol. 27, nº 2, 2008, p. 447-457, DOI : 10.1038/sj.emboj.7601976.

[10] Collet C., Thomassin J.-L., Francetic O., Genevaux P. et Nhieu G.T.V., « Protein polarization driven by nucleoid exclusion of DnaK(HSP70)-substrate complexes », Nature Communications, vol. 9, no 1, 2018, p. 2027, DOI : 10.1038/s41467-018-04414-2.

\section{PuBlications}

Thomassin J.-L., Santos Moreno J., Guilvout I., Tran Van Nhieu G. et Francetic O., «The trans-envelope architecture and function of the type 2 secretion system: new insights raising new questions », Molecular Microbiology, vol. 105, n 2, 2017, p. 211-226, DOI : 10.1111/mmi.13704.

Collet C., Thomassin J.-L., Francetic O., Genevaux P. et Tran Van Nhieu G., « Protein polarization driven by nucleoid exclusion of DnaK(HSP70)-substrate complexes », Nature Communications, vol. 9, no 1, 2018, p. 2027, DOI : 10.1038/s41467-018-04414-2.

Smail Y., Sun C., Combettes L. et Tran VAn Nhieu G., «Imaging $\mathrm{Ca}^{2+}$ responses during Shigella infection of epithelial cells », Journal of Visualized Experiments, $\mathrm{n}^{\mathrm{o}}$ 135, 2018, DOI : $10.3791 / 57728$.

Valencia-Gallardo C., Bou-Nader C., Aguilar D., Carayol N., Quenech'Du N., PeCQueur L., PARK H., FonteCAVE M., IZARD T. et NhIEU G.T.V., « Cell adhesion promoted by a unique Shigella IpaA vinculin- and talin-binding site », BioRxiv, 2018, 329136, DOI : $10.1101 / 329136$.

Tran Van Nhieu G., Dupont G. et Combettes L., « $\mathrm{Ca}^{2+}$ signals triggered by bacterial pathogens and microdomains », Biochimica Et Biophysica Acta. Molecular Cell Research, vol. 1865 , no 11 B, 2018, p. 1838-1845, DOI : 10.1016/j.bbamcr.2018.08.007.

\section{PHYSIQUE MULTI-ÉCHELLE DE LA MORPHOGÉNĖSE / MULTISCALE PHYSICS OF MORPHOGENESIS}

Responsables : Hervé TURLIER

\section{RECHERCHE}

Page web : https://www.college-de-france.fr/site/en-cirb/Turlier.htm.

\section{Introduction}

Invaluable progress has been made last decades in the molecular, genetic and cellular characterization of morphogenetic processes. Yet, the precise physical processes governing the shape and dynamics of cells remain poorly characterized. 